Despite the solid data, the model presented by McPheeters et al. is puzzling: why do vegetatively growing cells keep synthesizing mRNAs just to have them be degraded upon meiosis-triggering signals? As in some signaling pathways, such as oxidative stress, this may allow cells to quickly respond to damaging insults from the environment. However, it does seem energetically wasteful, especially if we consider that yeasts trigger meiosis when they sense poor nutrient conditions. A quick change in media composition to induce meiosis is something that happens only to yeast cultures growing in the laboratory, while in nature, yeast cells use up nutrients slowly as they grow, resulting in a very gradual change in medium composition.
From bacteria to mammalian cells, there are many mechanisms to avoid overlapping expression of different gene clusters. For example, in Mycobacterium tuberculosis, 13 different $\sigma$ subunits of RNA polymerase have been described to provide specificity to the transcription of gene clusters required to respond to different environmental inputs ${ }^{8}$. Eukaryotic cells have added several layers of complexity to this mechanism, regulating the differentiation pathways not only at the level of transcription. So far, these new mechanisms are shedding light on the molecular mechanisms underlying gene regulation during meiosis in fission yeast. But we can be assured that other processes will participate in this complex network.

\section{ACKNOWLEDGMENTS}

Work in the Ayté laboratory is supported by grants from the Ministerio de Ciencia e Innovación,Spain (BFU2006-01785) and the Consolider-Ingenio (2007-0020)

1. Mata, J., Lyne, R., Burns, G. \& Bahler, J. Nat. Genet 32, 143-147 (2002).

2. Mata, J., Wilbrey, A. \& Bahler, J. Genome Biol. 8, R217 (2007).

3. Harigaya, Y. et al. Nature 442, 45-50 (2006).

4. Moldon, A. et al. Nature 455, 997-1000 (2008).

5. McPheeters, D.S. et al. Nat. Struct. Mol. Biol. 16 , 255-264 (2009).

6. Averbeck, N., Sunder, S., Sample, N., Wise, J.A. \& Leatherwood, J. Mol. Cell 18, 491-498 (2005).

7. Malapeira, J. et al. Mol. Cell. Biol. 25, 6330-6337 (2005).

8. Manganelli, R., Dubnau, E., Tyagi, S., Kramer, F.R. \& Smith, I. Mol. Microbiol. 31, 715-724 (1999).

\title{
Yeast as budding stem cells?
}

DNA replication is semiconservative, with each strand serving as a template for the synthesis of a complementary strand. Thus, after one round of replication and cell division, each daughter cell will have a DNA molecule consisting of an old strand and a newly synthesized one. Each of these strands will serve as a template in the next round of replication, to generate two DNA molecules with strands of different 'ages': one comprising an old, original strand paired to a newly minted one, and the other with the strand synthesized in the first round also paired to a newly synthesized strand. There is a chance that errors will arise during replication, and, if not repaired, such mutations will become fixed in the next round of replication and passed along to one of the daughter cells. Thus, the only strands that will be completely free of replication errors are the original ones (provided there are no recombination events). John Cairns proposed in 1975 that it would be advantageous for adult stem cells

in metazoans to retain one of the original strands for each of their DNA molecules (that is, chromosomes), to avoid the accumulation of mutations - a concept known as the 'immortal strand' hypothesis. Whether such asymmetric DNA segregation indeed occurs in adult stem cells has been the subject of debate, with evidence both for and against it. There has also been speculation as to how cells would be able to segregate the sister chromatids in a nonrandom fashion during mitosis. Chromosomal segregation is mediated by spindle microtubules attached to the kinetochore, a structure that forms transiently on top of the centromeric DNA, so it has been proposed that differences in this region could mark the chromosome containing the immortal strand.

Now Thorpe, Bruno and Rothstein find that four kinetochore components (Ndc10, Ctf19, Mtw1 and Ask1) are indeed segregated asymmetrically in postmeiotic budding yeast (Proc. Natl. Acad. Sci. USA, in the press, doi:10.1073/PNAS.0811248106). This unicellular organism undergoes asymmetric cell division, with one mother cell and one bud being generated at each cell division. The authors fused candidate kinetochore proteins to yellow or cyan fluorescent protein (YFP or CFP), made a diploid yeast strain containing both fusions, and then had those cells undergo meiosis to generate spores carrying the sequence for only one of the labeled constructs but containing both YFP- and CFP-fused proteins. The fate of the non-encoded protein as well as the encoded protein was then followed from the germinating spore through three generations via fluorescence microscopy.

In the first round of division, the fluorescence signal was on average twofold stronger in the mother cell than in the bud cell as shown in the microscopy images (mother and bud cells indicated by " $\mathrm{m}$ " and " $\mathrm{b}$ " on the top left, fluorescence in the bottom left, with an enhanced view on the right), indicating an asymmetric segregation of the kinetochore protein. In the next round of cell replication, a similar behavior was observed for the mother cell, whereas the previous "bud" cell segregated the protein equally between itself and its daughter bud. The same thing happened in the third round of cell division, thus defining a cell lineage or pedigree, as represented in the drawing on the right. Such an asymmetric segregation pattern was not observed with another nuclear protein, histone $\mathrm{H} 2 \mathrm{~A}$, nor was it seen in vegetatively growing cells.

Budding yeast cells have a limited reproductive lifespan, and the mother-cell lineage stops generating new daughters after a certain number of cellular divisions. In fact, the mother cells accumulate extrachromosomal DNA circles and oxidized proteins, which may contribute to ensuring a longer reproductive lifespan for the daughter bud cells. So one may ask, why would the postmeiotic cells studied here segregate their kinetochores, and hence their chromatids, in such an asymmetric way? It also remains to be shown whether sister chromatids are indeed segregated in a nonrandom fashion in the progeny of a germinating yeast spore. But regardless of 'why', this work opens new avenues to explore 'how' asymmetric segregation can occur. It also shows that budding yeast, the geneticists' darling organism, can be a valuable model for studying the establishment of cellular lineages and asymmetric cell division in adult stem cells.

Inês Chen 